\title{
SECOND ORDER ELLIPTIC EQUATIONS WITH MIXED BOUNDARY CONDITIONS
}

\author{
BY A. J. PRYDE \\ Communicated by C. Davis, October 27, 1976
}

We consider the mixed boundary value problem (MBVP) $A u=f$ in $\Omega$, $B^{+} u=g^{+}$in $\Gamma^{+}, u=g^{-}$in $\Gamma^{-}$where $\Omega$ is a bounded open subset of $R^{n}$ whose boundary $\Gamma$ is divided into disjoint open subsets $\Gamma^{+}$and $\Gamma^{-}$by an $(n-2)$-dimen. sional manifold $\omega$ in $\Gamma$. We assume $A=\Sigma_{|\alpha| \leqslant 2} a_{\alpha}(x) D^{\alpha}$ is a properly elliptic operator on $\bar{\Omega}$ and $B^{+}=\sum_{j=1}^{n} b_{j}^{+}(x) D_{j}+b_{0}(x)$ is a normal boundary operator satisfying the complementing condition with respect to $A$ on $\overline{\Gamma^{+}}$. The coefficients of the operators and $\Gamma^{+}, \Gamma^{-}$and $\omega$ are all assumed arbitrarily smooth.

Throughout, $s$ will denote a real number with $s \neq 1 / 2(\bmod 1)$. For $G=R^{n}$, $R_{ \pm}^{n}, \Omega$ or $\Gamma$, the Sobolev spaces $H^{s}(G)$ are as in Lions-Magenes [1]. Also $H^{s}\left(\Gamma^{ \pm}\right)$ is the space of restrictions to $\Gamma^{ \pm}$of distributions in $H^{s}(\Gamma)$, with the infimum norm, and $H_{A}^{s}(\Omega)=\left\{u \in H^{s}(\Omega): A u \in L^{2}(\Omega)\right\}$ with the graph norm. Let $\gamma_{0}$ : $H_{A}^{s}(\Omega) \rightarrow H^{s-1 / 2}(\Gamma)$ be the trace map, $r^{ \pm}: H^{s-1 / 2}(\Gamma) \rightarrow H^{s-1 / 2}\left(\Gamma^{ \pm}\right)$the restriction maps, and $\gamma^{-}=r^{-} \gamma_{0}$. Then $B^{+}=r^{+} B$ for some first-order normal boundary operator $B$ on the whole of $\Gamma$.

Consider the maps $\left(A, \gamma^{-}, B^{+}\right)_{s}$ defined as

$$
\begin{array}{ll}
\left(A, \gamma^{-}, B^{+}\right): H^{s}(\Omega) \rightarrow H^{s-2}(\Omega) \times H^{s-1 / 2}\left(\Gamma^{-}\right) \times H^{s-3 / 2}\left(\Gamma^{+}\right) & \text {if } s>3 / 2, \\
\left(A, \gamma^{-}, B^{+}\right): H_{A}^{s}(\Omega) \rightarrow L^{2}(\Omega) \times H^{s-1 / 2}\left(\Gamma^{-}\right) \times H^{s-3 / 2}\left(\Gamma^{+}\right) & \text {if } s<3 / 2 .
\end{array}
$$

These maps are bounded for all $s$, by the condition of normality for $s<3 / 2$ (see for example $[1, \S 2.8 .1])$. The MBVP is called well-posed if there exists $s \neq 1 / 2(\bmod 1)$ for which $\left(A, \gamma^{-}, B^{+}\right)$, is Fredholm. A bounded linear operator between Hilbert spaces is called $\alpha$-semi-Fredholm $(\alpha \mathrm{sF})$ if it has finite dimensional kernel and closed range, $\beta$-semi-Fredholm $(\beta \mathrm{sF})$ if it has closed range with finite codimension, and Fredholm if it is $\alpha \mathrm{sF}$ and $\beta \mathrm{sF}$.

THEOREM. For each $x \in \omega$ there is an open subset $I_{x}$ of the reals such that for $s \neq 1 / 2(\bmod 1),\left(A, \gamma^{-}, B^{+}\right)_{s}$ is Fredholm if and only if $s \in I=\bigcap_{x \in \omega}$ $I_{x}$. Moreover, $I$ is open and so the MBVP is well-posed if and only if I is nonempty. In fact, for each $x \in \omega$ there is a real number $e_{x}$ determined algebraically

AMS (MOS) subject classifications (1970). Primary 35J 20, 35J25, 47F05.

Key words and phrases. Mixed boundary value problem, properly elliptic operator, Sobolev space, Fredholm operator, well-posed problem, sesquilinear form, spaces with homogeneous norms, Wiener-Hopf operator. 
by the coefficients of $A$ and $B^{+}$at $x$ such that-with $e=\left\{r \in \mathbf{R}: r \equiv e_{x}(\bmod 1)\right.$ for some $x \in \omega\}$ :

(a) if $n=2, I_{x}=\left\{r \in \mathbf{R}: r \neq e_{x}(\bmod 1)\right\}$ and, hence, $I=R-e$;

(b) if $n=3, I_{x}=\left(e_{x}, e_{x}+1\right)$ or $I_{x}=\varnothing$, and $\left(A, \gamma^{-}, B^{+}\right)_{s}$ is $\alpha s F(\beta s F)$ if and only if $s \notin e$ and $s>\sup _{x \in \omega} e_{x}\left(s<\inf _{x \in \omega} \tilde{e}_{x}\right)$ where $\tilde{e}_{x}$ is $e_{x}+1$ in the first case, and $e_{x}$ in the second;

(c) if $n>3, I_{x}=\left(e_{x}, e_{x}+1\right)$ and $\left(A, \gamma^{-}, B^{+}\right)_{s}$ is $\alpha s F(\beta s F)$ if and only if $s \notin e$ and $s>\sup _{x \in \omega} e_{x}\left(s<\inf _{x \in \omega} e_{x}+1\right)$.

Peetre [2] showed that $\left(A, \gamma^{-}, B^{+}\right)_{s}$ is Fredholm for $s>3 / 2$ and $s \notin e$, without the assumption of normality, but only for $n=2$. Shamir [5] and Visik and Eskin [6] provided elements of the solution of the MBVP for $n>2$, but the restriction $s>3 / 2$, required for nonnormal problems, and in addition, the problems of localising when $s<3 / 2$, prevented them from finding necessary and sufficient conditions for the problem to be well posed in our sense.

For normal $B^{+}$, we can construct a bounded sesquilinear form $J^{s}[u, v]$ on a closed subspace $V^{s}(\Omega) \times W^{2-s}(\Omega)$ of $H^{s}(\Omega) \times H^{2-s}(\Omega)$ such that $\left(A, \gamma^{-}, B^{+}\right)_{s}$ is $\alpha s \mathrm{~F}(\beta s \mathrm{~F})$ if and only if $J^{s}$ is $\alpha s \mathrm{~F}(\beta s \mathrm{~F})$ in the sense that the operator $T^{s}: V^{s}(\Omega) \rightarrow W^{2-s}(\Omega)^{*}$ defined by $\left\langle T^{s} u, v\right\rangle=J^{s}[u, v]$ is $\alpha \mathrm{sF}(\beta s \mathrm{~F})$. This result is an easy consequence of Pryde [3].

By Peetre's lemma [1, Lemma 2.5.1] $J^{s}$ is $\alpha \mathrm{SF}$ if and only if

$$
\|u\|_{V^{s}(\Omega)} \leqslant c\left(\sup _{v \in W^{2-s(\Omega)}} \frac{\left|J^{s}[u, v]\right|}{\|v\|_{W^{2-s}(\Omega)}}+\|u\|_{H^{s-1}(\Omega)}\right), \quad u \in V^{s}(\Omega),
$$

and $\beta \mathrm{sF}$ if and only if

(2) $\|v\|_{W^{2-s}(\Omega)} \leqslant c\left(\sup _{u \in V^{s}(\Omega)} \frac{\left|J^{s}[u, v]\right|}{\|u\|_{V^{s}(\Omega)}}+\|v\|_{H^{1-s}(\Omega)}\right), \quad v \in W^{2-s}(\Omega)$.

The advantage of looking at forms is that their estimates can be localised for all $s$. For this we use spaces with homogeneous norms, $Z^{s}(G)$ and $\AA^{s}(G)$ for $G=\mathbf{R}^{n}$ or $\mathbf{R}_{ \pm}^{n}$. In fact, let $[u ; G]_{s}$ be the norm on $C_{0}^{\infty}(\bar{G})$ defined by

(i) if $s=0,[u ; G]_{s}=\|u\|_{L^{2}(G)}$;

(ii) if $0<s<1,[u ; G]_{s}=\left(\int_{G} \int_{G}|u(x)-u(y)|^{2} /|x-y|^{n+2 s} d x d y\right)^{1 / 2}$;

(iii) if $s \geqslant 1,[u ; G]_{s}=\left(\Sigma_{|\alpha|=[s]}\left[D^{\alpha} u ; G\right]_{s-[s]}^{2}\right)^{1 / 2}$ where [s] is the integral part of $s$.

For $s \geqslant 0, Z^{s}(G)\left(Z^{s}(G)\right)$ is the completion of $C_{0}^{\infty}(\bar{G})\left(C_{0}^{\infty}(G)\right)$ with respect to $[u ; G]_{s}$. For $s<0, Z^{s}(G)\left(\mathcal{Z}^{s}(G)\right)$ is the strong dual of $\mathcal{Z}^{-s}(G)\left(Z^{-s}(G)\right)$.

These spaces have been considered before, but their theory not fully developed. See, for example, Shamir [4]. They are the natural spaces for boundary value problems in $\mathbf{R}_{+}^{n}$ with homogeneous operators and constant coefficients.

For each $x \in \omega$, we freeze the coefficients of $A$ and $B^{+}$at $x$, and drop all lower order terms, obtaining homogeneous operators with constant coefficients $A_{x}$ and $B_{x}^{+}$. Define $\left(\gamma^{-}, B^{+}\right)_{s, x}$ to be 


$$
\left(\gamma^{-}, B_{x}^{+}\right): Z_{\mathrm{ker} A_{x}}^{s}\left(\mathbf{R}_{+}^{n}\right) \rightarrow Z^{s-1 / 2}\left(\mathbf{R}_{-}^{n-1}\right) \times Z^{s-3 / 2}\left(\mathbf{R}_{+}^{n-1}\right)
$$

where $X_{\text {ker } A}$ denotes the kernel of an operator $A$ in the space $X$.

Using the results of Pryde [3] repeatedly, and the usual localisation techniques, it follows that $J^{s}$ is $\alpha \mathrm{sF}(\beta \mathrm{sF})$ if and only if $\left(\gamma^{-}, B^{+}\right)_{s, x}$ is left invertible (onto) for each $x \in \omega$.

Following Peetre [2], we then construct certain Wiener-Hopf operators $r^{+} M_{x}^{s}: L^{2}\left(\mathbf{R}_{+}^{n-1}\right) \rightarrow L^{2}\left(\mathbf{R}_{+}^{n-1}\right)$ whose symbols are determined by the coefficients of $A_{x}$ and $B_{x}$. It follows that $\left(\gamma^{-}, B^{+}\right)_{s, x}$ is left invertible (onto) if and only if $r^{+} M_{x}^{s}$ is left invertible (onto).

Finally, using the results of Shamir [5], we find real numbers $e_{x}, \tilde{e}_{x}$ and open sets $I_{x}$ as above, such that $r^{+} M_{x}^{s}$ is an isomorphism if and only if $s \in I_{x}$. Moreover, if $n \geqslant 3, r^{+} M_{x}^{s}$ is left invertible (onto) if and only if $s>e_{x}\left(s<{\widetilde{e_{x}}}_{x}\right)$ and $s \neq e_{x}(\bmod 1)$. If $n>3, \tilde{e}_{x}=e_{x}+1$ and if $n=3, \tilde{e}_{x}=e_{x}+1$ or $e_{x}$.

Detailed proofs and corresponding results for higher order operators will appear in another paper. The work was part of a $\mathrm{Ph} . \mathrm{D}$. thesis at Macquarie University. I am indebted to my supervisor Alan McIntosh.

\section{REFERENCES}

1. J. L. Lions and E. Magenes, Nonhomogeneous boundary value problems and applications, Vol. 1, Dunod, Paris, 1968; English transl., Springer-Verlag, Berlin and New York, 1972. MR $40 \# 512 ; 50 \# 2670$.

2. J. Peetre, Mixed problems for higher order elliptic equations in two variables. I, II, Ann. Scuolo. Norm. Sup. Pisa (3) 15 (1961), 337-353; ibid. 17 (1963), 1-12. MR 25 \#4240; 28 \#328.

3. A. J. Pryde, The five lemma for Banach spaces, Proc. Amer. Math. Soc. (to appear).

4. E. Shamir, Mixed boundary value problems for elliptic equations in the plane. The ${ }_{L}^{p}$ theory, Ann. Scuola Norm. Sup Pisa (3) 17 (1963), 117-139. MR 28 \#329.

5. Multipliers of Fourier transform in a half-space, Bull. Amer. Math. Soc. 71 (1965), 165-167. MR 30 \#2296.

6. M. I. Višik and G. I. Éskin, Equations in convolution in a bounded region, Uspehi Mat. Nauk 20 (1965), 89-152 = Russian Math. Surveys 20 (1965), 85-151. MR 32 \#2741.

SCHOOL OF MATHEMATICS AND PHYSICS, MACQUARIE UNIVERSITY, NORTH RYDE, N.S.W., 2113, AUSTRALIA 